\title{
A FUZZY HOMOMORPHIC ALGORITHM FOR IMAGE ENHANCEMENT
}

\author{
U. A. Nnolim* \\ DEPARTMENT OF ELECTRONICS ENGINEERING, UNIVERSity OF NigERIA, NSUKKA, ENUGU STATE-NIGERIA \\ E-mail Address: uche.nnolim@unn.edu.ng
}

\begin{abstract}
The implementation and analysis of a novel Fuzzy Homomorphic image enhancement technique is presented. The technique combines the logarithmic transform with fuzzy membership functions to deliver an intuitive method of image enhancement. This algorithm reduces the computational complexity by eliminating the need for image-sizedependent filter kernels and the forward and inverse Fourier Transforms. The proposed algorithm is compared with the more established algorithms for the enhancement of low contrast images with uneven illumination. The results show that the fuzzy method provides similar or better results than the frequency domain method and some other well-known image enhancement algorithms.
\end{abstract}

Keywords—Homomorphic Filtering, Illumination correction, Low Contrast Images, Enhancement, Filtering, Fuzzy Logic

\section{INTRODUCTION}

The Homomorphic filtering method is a well-known technique for image enhancement and has been employed in various applications [1, 2]. It produces simultaneous dynamic range compression and contrast enhancement [3-6]. The prevalent form of the algorithm is performed in the frequency domain. However, the frequency transform-based method is hard and crisp method in that parameters are inflexible. This makes it hard to tune and not very intuitive. Fuzzy logic and fuzzy set theory has been widely used in image processing areas such as image restoration and contrast enhancement [7-14]. However, there is no specific application to date of fuzzy methods for image enhancement techniques such as Homomorphic filtering. However, some work in the literature has incorporated a Fuzzy rule-based approach to the problem of dynamic range compression and contrast enhancement [5]. A novel Fuzzy Homomorphic image enhancement technique is proposed. The proposed technique avoids the use of filtering masks by performing point operations in the Fuzzy domain.

A brief review of classical Homomorphic filtering is presented in Section II followed by the implementation and analysis of the Fuzzy Homomorphic method in Section III. Section IV provides results for the proposed method, which are compared with other image enhancement algorithms.

\section{ANALYSIS OF HOMOMORPHIC FILTERING}

The mathematical principle behind homomorphic filtering theory is rooted in the illuminationreflectance approximation model of image scenes [4]. In this model, the image, $z(x, y)$, is the product of the illumination, $i(x, y)$ (which is comprised of low frequency components) and the reflectance, $r(x, y)$ (high frequency components) [6]. The image is expressed as:

$z(x, y)=i(x, y) * r(x, y)$

The illumination is responsible for the dynamic range of the image scene while the reflectance determines the level of detail (contrast) [5, 6]. The assumption is that the illumination component varies slowly, unlike the reflectance component. This slow spatial variation in the illumination component is the reason for its association with the low frequency components [15] and makes dynamic range compression possible.

The frequency domain Homomorphic filtering technique is mathematically defined in (2);

$$
z^{\prime}(x, y)=e^{\left(\mathcal{F}^{-1}\left\{\mathcal{F}\{\ln |z(x, y)|\} * H_{H P F}(u, v)\right\}\right)}
$$

In (2) $H_{H P F}(u, v)$ is the frequency domain high pass filter (HPF), while $\mathrm{u}$ and $\mathrm{v}$ are the frequency coordinates. The symbols $\mathcal{F}$ and $\mathcal{F}^{-1}$ are the Fourier 
Transform and Inverse Fourier Transform operators respectively. The image in the frequency domain is non-separable due to the multiplicative nature of the illumination and reflectance components [4]. The logarithm transform converts the multiplicative/ nontrivial result of the reflectance and illumination components to an additive/trivial one $[6,15]$. The logarithmic transformation is mathematically defined as;

$$
\begin{aligned}
& \ln |z(x, y)|=\ln |i(x, y) * r(x, y)|= \\
& \ln |i(x, y)|+\ln |r(x, y)|
\end{aligned}
$$

After the Logarithmic Transformation shown in Eqn (3), the Fourier Transformation of the imagecan then be performed. Figure 1 shows the Homomorphic filtering operation expressed in Eqn (2).

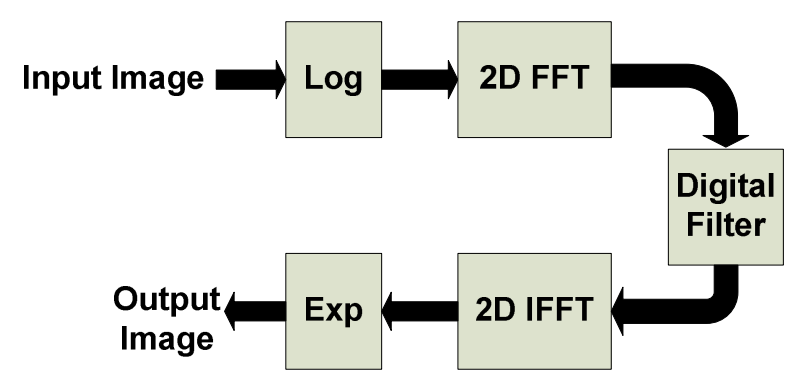

Figure1. Frequency Domain Homomorphic Filtering Technique

\section{FUZZY HOMOMORPHIC IMAGE ENHANCEMENT}

After the conversion of the multiplicative nature of the illumination and reflectance component of an image to a linear, additive form, it is easy to transform the log image into the fuzzy domain using a suitable membership function. In Figure 2, a Fourier transformation of the logarithm-transformed image is performed to aid in the visualization of high and low frequency regions of the image. The linearization effect of the logarithm operator is observed, with regards to the image

If a brightness attribute is defined according to the level of increased detail, high frequency variation or contrast enhancement, the fuzzy process becomes intuitive as follows; the fuzzification of the log image translates the low frequency values into values with zero or near zero membership in the fuzzy domain of the attribute brightness while the high frequency values are then defined as values with full or near full membership of the brightness attribute.

This can be observed in Figure 3. Within this new domain, image enhancement becomes a simple exercise of modifying the membership values using an appropriate function $[8,10,11,14]$. The proposed new system for the image enhancement is as shown in Figure 4.

The choice of the membership function is vital to the effectiveness of the enhancement technique [11, 14]. After experimenting with a series of membership functions, linear or almost linear membership functions produced the best results. Based on experimental image results, linear fuzzy membership functions are well-suited to the fuzzification of the log image. An initial membership function used was the typical function used in the fuzzy intensification (INT) operator $[8,11,13,14,16]$. The function is defined as:

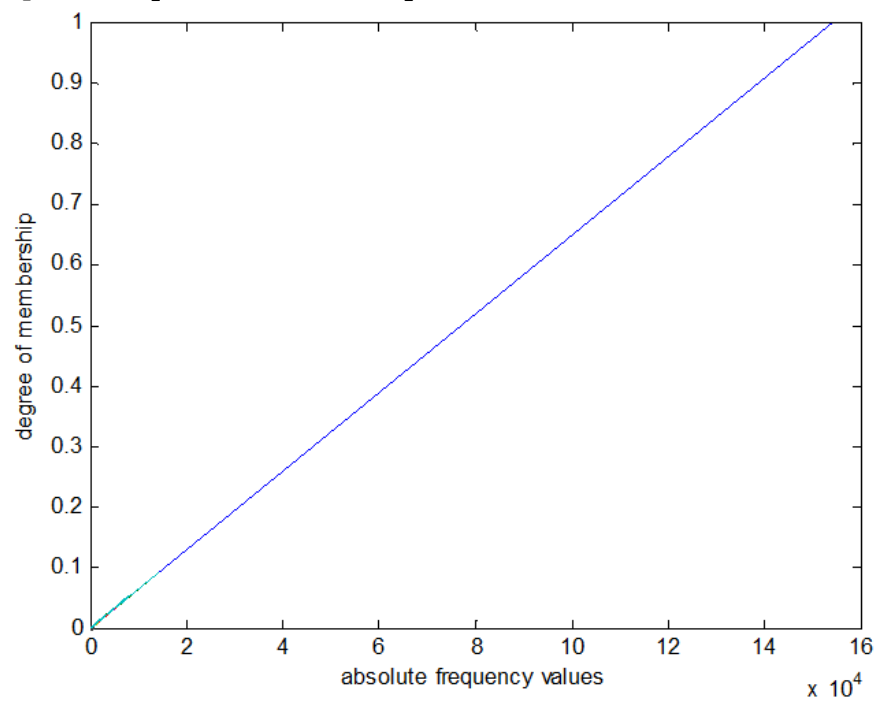

Figure 2. Fuzzy Membership of Fourier Transformed Logarithmic Image

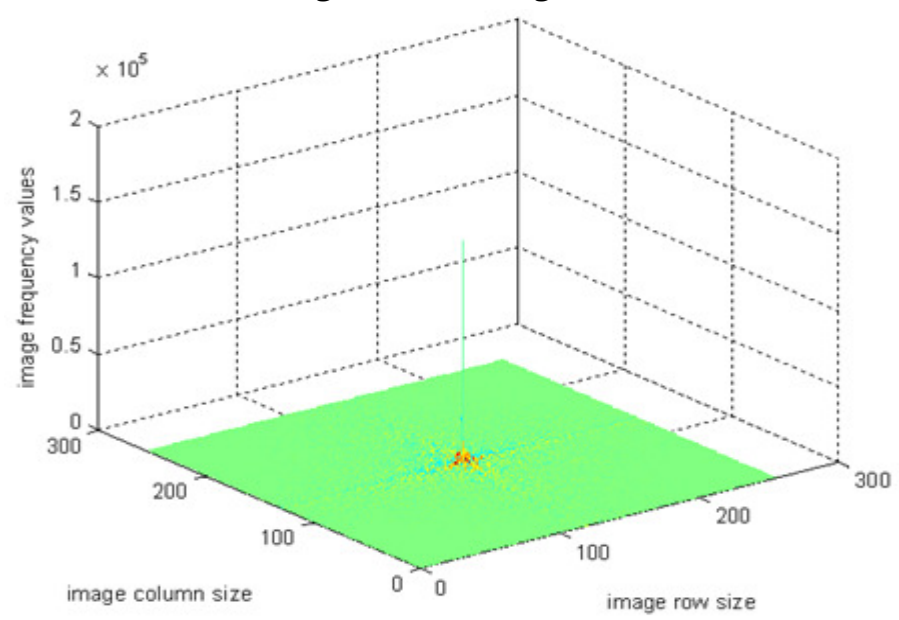

Figure3. Fourier transformed logarithmic image

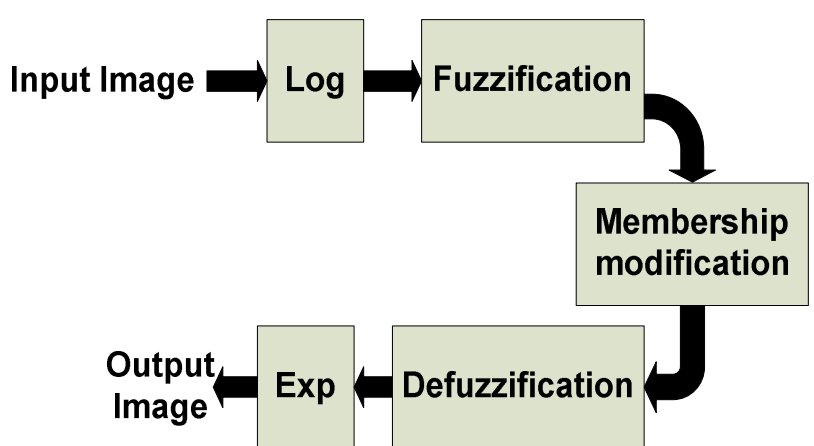

Figure 4. Fuzzy Homomorphic Image Enhancement 


$$
\mu_{m n}=\left[1+\frac{\left[g_{\max }-g_{m n}\right]}{F_{d}}\right]^{-F_{e}}
$$

In Eqn. (4), $g_{m n}$ is the gray level of the image at coordinates $(m, n)$ and $\mu_{m n}$ is the membership function value of the fuzzified image at position $(m, n)$ and $F_{e}=2$ while

$$
F_{d}=\frac{g_{\max }-g_{\text {mid }}}{\left(0.5^{-F e}\right)-1}, \text { where } g_{\text {mid }}=\frac{g_{\max }+g_{\min }}{2}
$$

However, the function in Eqn. (4) doesn't utilize the full range (0 to 1$)$ of membership since the minimum value in the fuzzified image is not zero (clearly seen in Figure 5).

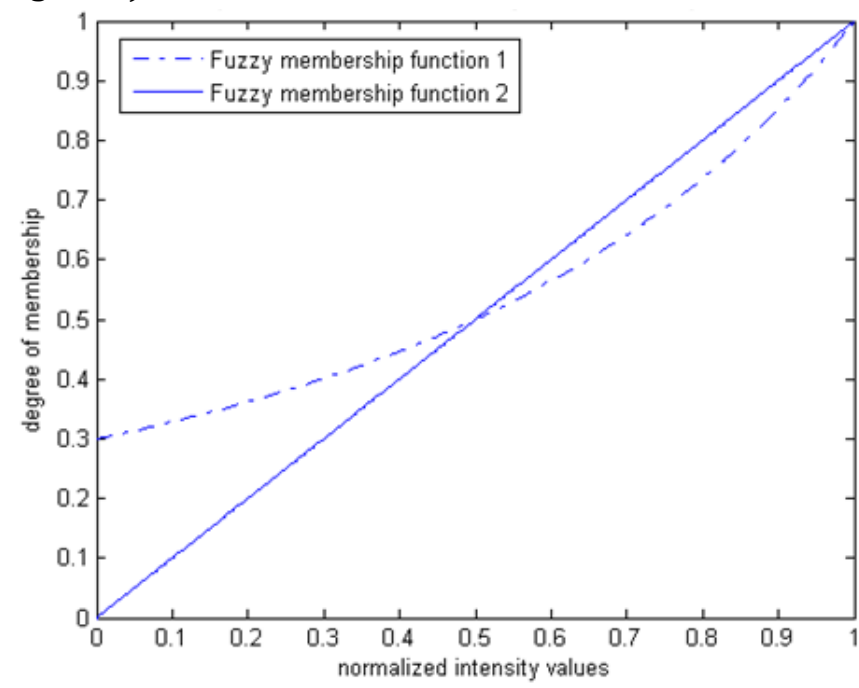

Figure 5. Mapping of logarithmic values of intensity values to fuzzy domain using fuzzy Membership Functions 1 and 2

Thus any modification of this membership function leads to unsatisfactory results. A more suitable linear membership function [14] was employed and is defined as;

$$
\mu_{m n}=\frac{g_{m n}-g_{\min }}{g_{\max }-g_{\min }}
$$

The results of using this membership function can be seen in Figure 6 when compared with the previous membership function. For ease of interpretation, the membership function in (4) is designated as Membership Function 1 while the equation in (5) is Membership Function 2. Figs. 5 and 6 show the mapping of the intensity values for images with 256 levels of gray and the logarithm of the intensity values respectively.

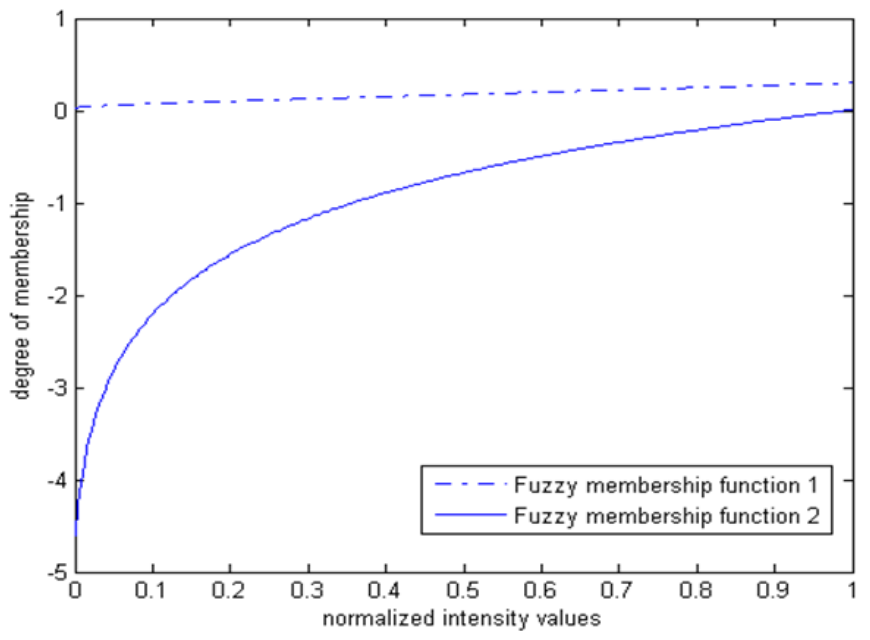

Figure 6. Fuzzified logarithms of intensity values (256 levels of gray)

The next crucial task is to select an appropriate membership function to be employed in the modification of membership.

For membership modification, the function must be able to moderately modify (increase or decrease) the mid-range membership while drastically modifying the lowest membership values and leaving the highest membership values unchanged. A custom membership function used at the modification stage is defined as;

$\mu_{m n}^{\prime}=1-\left[\left(\frac{N-1}{N}\right)\left(1-\mu_{m n}\right)^{\beta}\right]$, where $N \geq 2$

In (7), $\beta$ is a linguistic hedge $[13,14]$ signifying degree of brightness while $N$ acts as a membership modifier/boost ratio analogous to the high frequency multiplier (boost factor) $[3,4]$ in the conventional Homomorphic filter, which controls the level of enhancement of the high frequency components (reflectance values) and the attenuation of the low frequency components (illumination component) of the image. In the fuzzy domain, $N$ determines the percentage increase in membership of the brightness attribute for membership values close to zero, which represent the low frequency values in the image. The membership values close to 1 are unchanged. Based on experiments, a good value for $N$ is found to be usually 4. Fuzzy methods provide an intuitive method of image enhancement and this is observed in the algorithm. A plot of the membership function as a function of $N$ is shown in Figure 7. In the right-hand part of Figure 7, the plot is magnified for clarity. 

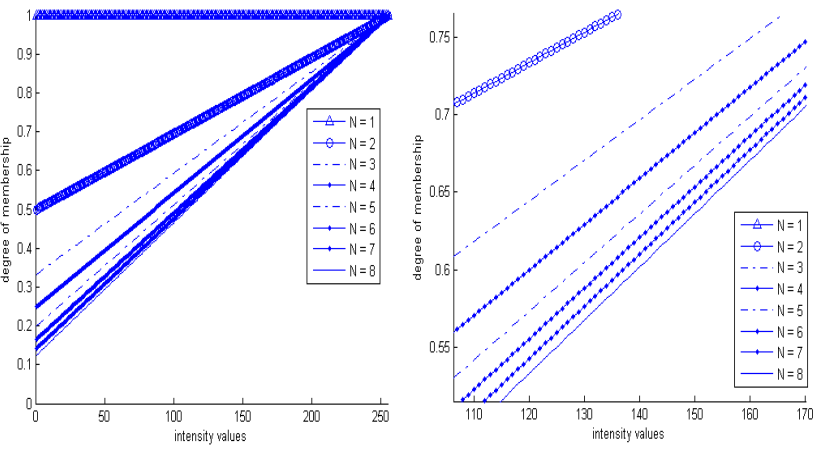

Figure 7 Fuzzy membership function for varying levels of $N$

The vertical axis represents the degree of membership while the horizontal axis represents the intensity values observed over varying values of $N$.

\section{EXPERIMENTAL RESULTS}

The Fuzzy Homomorphic algorithm was employed in the enhancement of low contrast test images [14] with uneven illumination and the results are presented in this section. Fig 8 shows a sample of the images tested with the proposed algorithm. For all image enhancement experiments, the fuzzy hedge, $\beta=2$ and $N=4$.

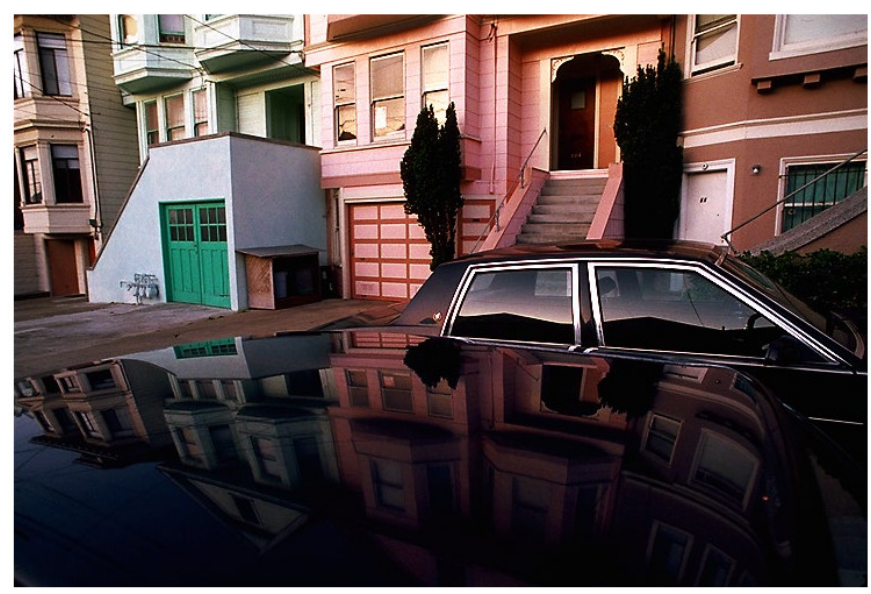

(a)

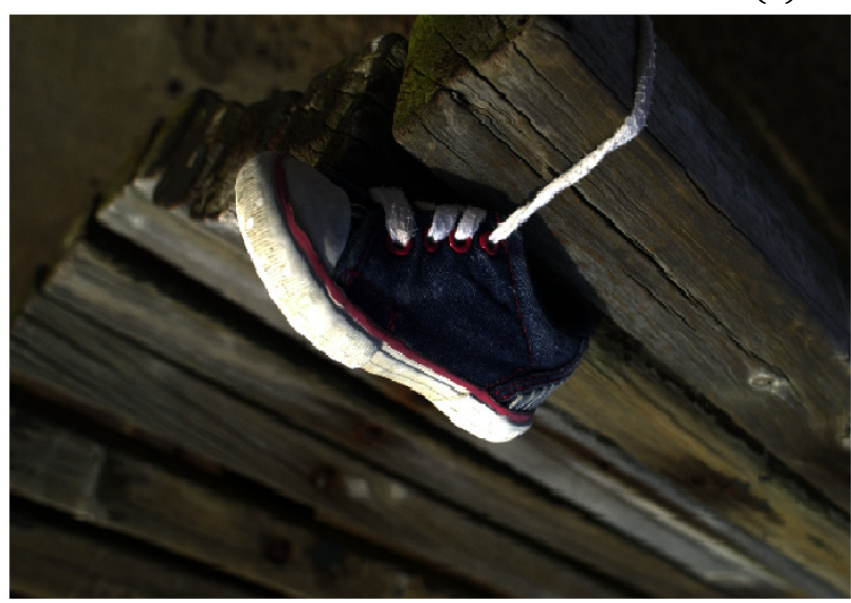

(c)
In Figure 9, the initial and modified membership functions of the fuzzified shoe image are shown for comparison. The graph is basically a plot of the membership degree versus the actual image.
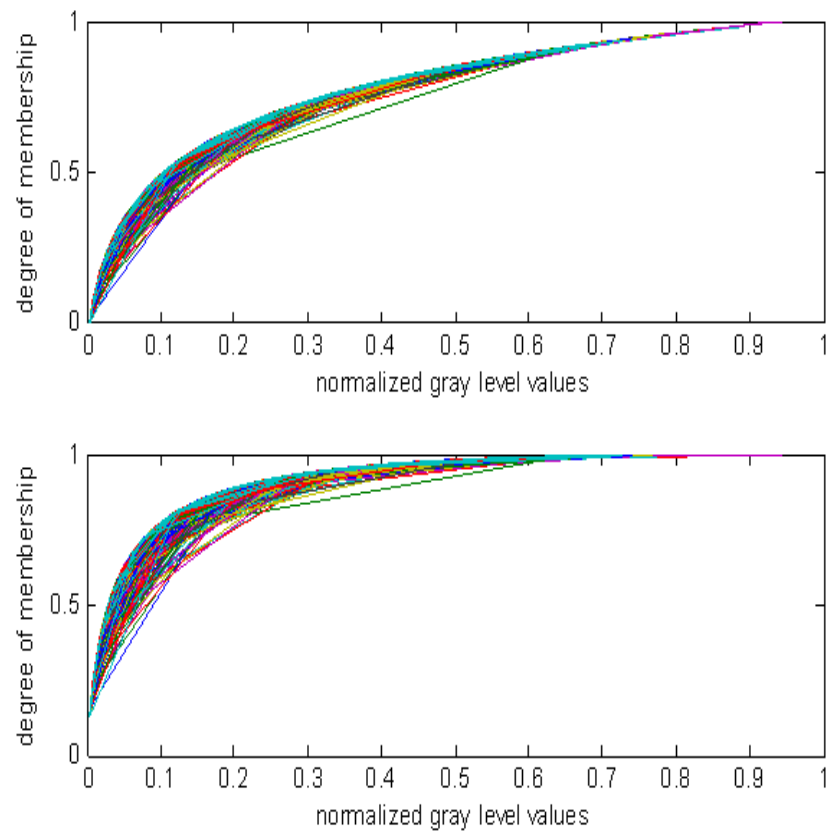

Figure 9. Plot of original and modified image gray level values in the fuzzy domain against fuzzy membership

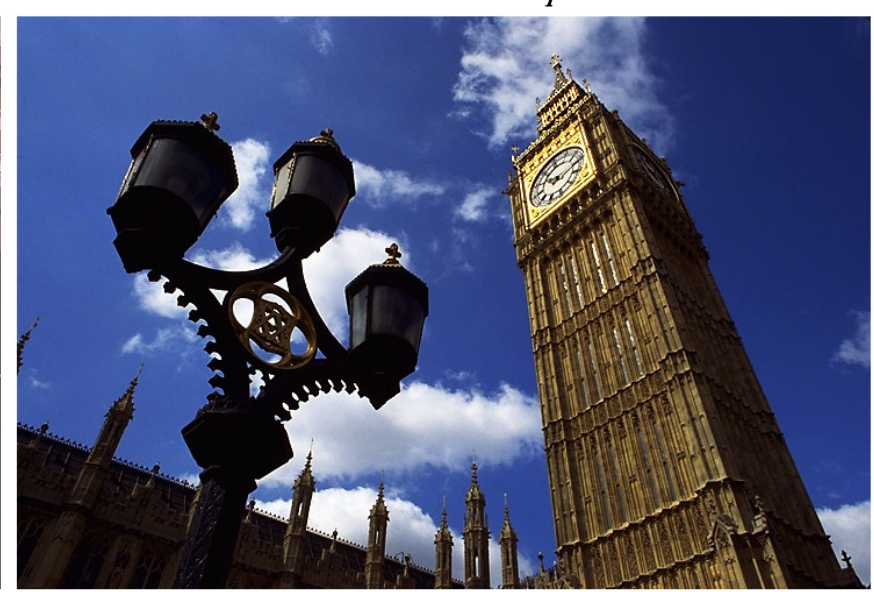

(b)

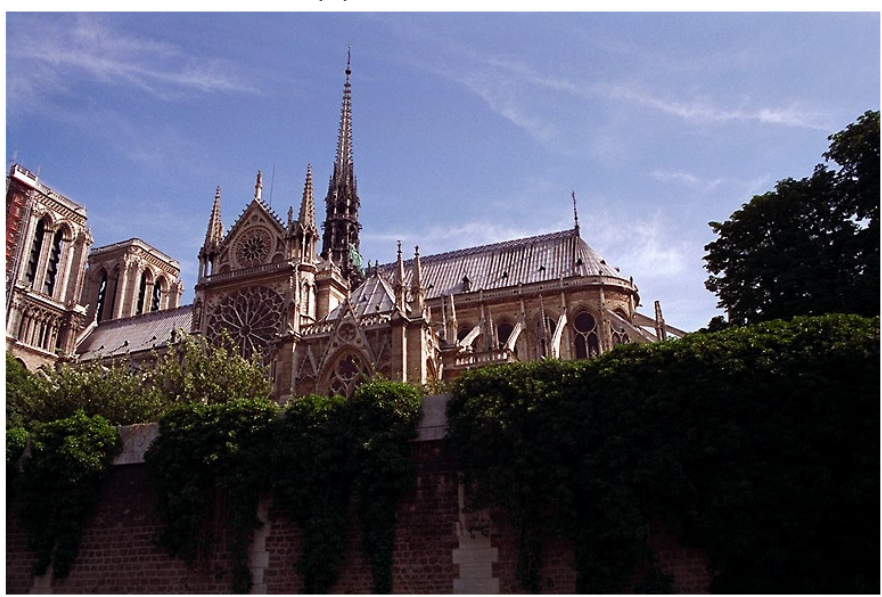

(d)

Figure 8. Original image test images(a) car image (b) tower clock image (c) shoe image and (d) cathedral image 
The membership function in (6) provides a soft result in that in certain images, differences in pixel values and their neighbours lead to non-uniformity in certain images. However, multiple iterations attempt to correct for this. Also very dark images may require multiple iterations as well. Based on current experiments, only 2 iterations are required. However, multiple iterations lead to the enhancement of JPEG artifacts forcertain compressed images.

The proposed method is provided in comparison with the conventional Homomorphic filtering method, Multi Scale Retinex with Colour Restoration (MSRCR) [17] and Histogram Equalization (HE) as shown in Figure 10.

The results were evaluated using fuzzy and crisp statistical measures for quantitative comparison. The fuzzy measures include the Fuzzy Entropy (FE), Linear Fuzziness Index (LFI) and Quadratic Fuzziness Index (QFI) $[13,14,18]$, which are defined as;

$$
\begin{gathered}
F E=\frac{-1}{L \ln 2(M N)} \sum_{x=0}^{L-1}[\mu(x) \ln (\mu(x))+(1- \\
\mu(x)) \ln (1-\mu(x))] h(x) \\
L F I=-\frac{2}{M N} \sum_{x=0}^{L-1}[h(x) \min (\mu(x), 1-\mu(x))] \\
Q F I=\frac{2}{\sqrt{M N}} \sum_{x=0}^{L-1}\left[h(x)(\min (\mu(x), 1-\mu(x)))^{2}\right]^{0.5}
\end{gathered}
$$

Where $h(x)$ is the image histogram, $M$ and $N$ are the row and column dimensions of the image, $L$ is the number of gray levels and $\mu(x)$ is the fuzzy membership function.

The defined fuzzy image quality measures in (8) to (10) are suited to image enhancement since the judgment of quality is not a crisp measure. The index of fuzziness describes how fuzzy the image is. Maximum fuzziness occurs when the index of fuzziness is 0.5 , meaning that contrast is effectively zero. The fuzzy entropy describes the amount of fuzzy information that can be extracted from the image.

The statistical measures used are the standard deviation and variance. The standard deviation measures the spread of the distribution. Thus images with more contrast are more spread out in terms of their histogram distribution and have higher standard deviation. The variance is the square of the standard deviation and these two quantities are invariably related to contrast. However, due to the lack of a reference image for comparison, a derived term of measurement is used. These are used as relative values composed of ratios to measure relative contrast enhancement. It is defined simply as a measure of the ratios of the final to initial variance (relative variance, RV) and standard deviations of the images (relative standard deviation, RSD).

For the measurements of quantities, the intensity information was decoupled from the Saturation and Hue quantities using the HSI colour space [3]. This enables easier understanding of the intensity information unlike in the RGB colour model where each colour value is dependent on all of $R, G$ and $B$ values.

Higher FE values mean more membership values are closer to 1(brightness) while lower FE values mean more membership values are closer to 0 (darkness).

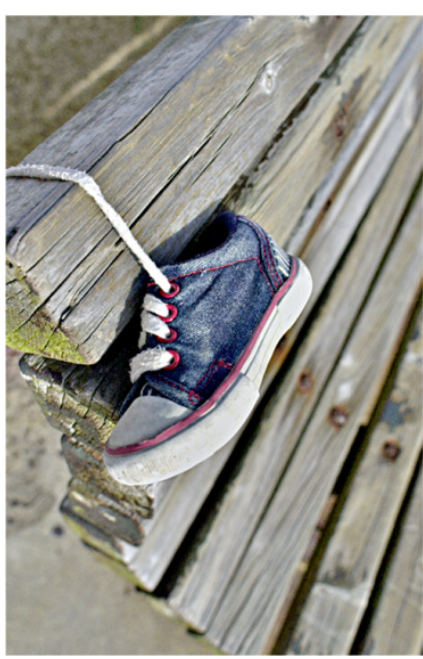

(a)

(c)

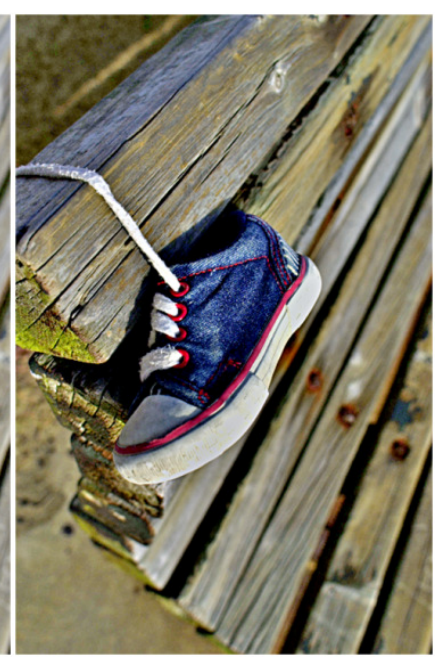

(b)

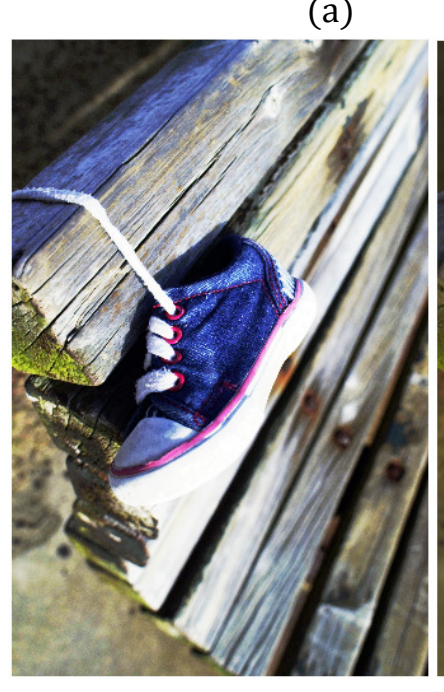

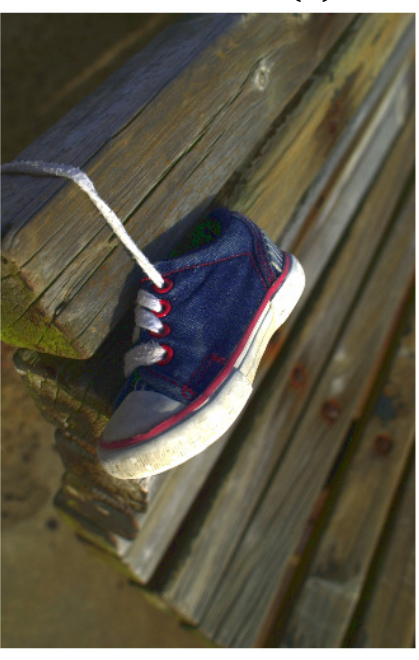

(d)
The fuzzy and crisp statistical parameters for the enhanced image using fuzzy Homomorphic algorithm, MSRCR, HE and classical Homomorphic filtering are given in Table I. For the original image, RV \& RSD values of $1 \mathrm{imply}$ that no enhancement is performed. 


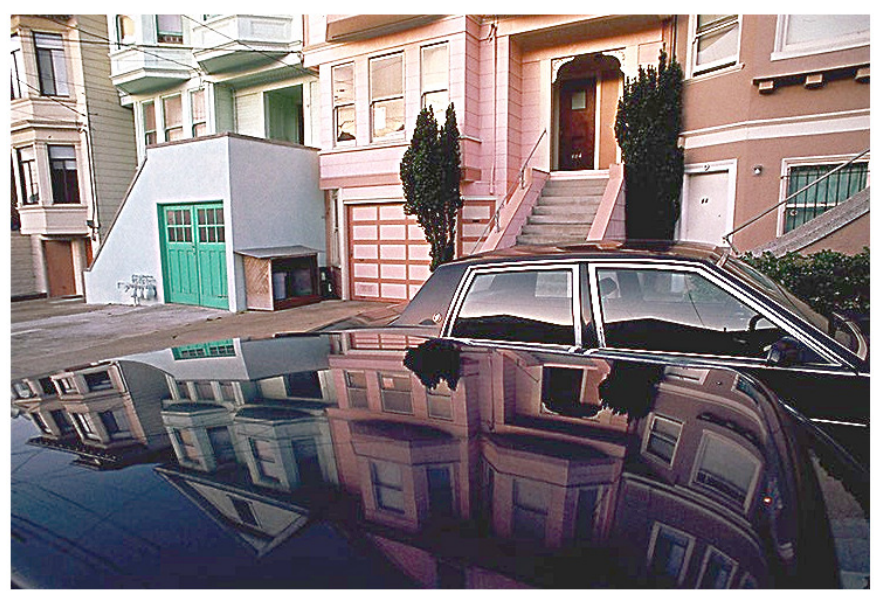

(a)

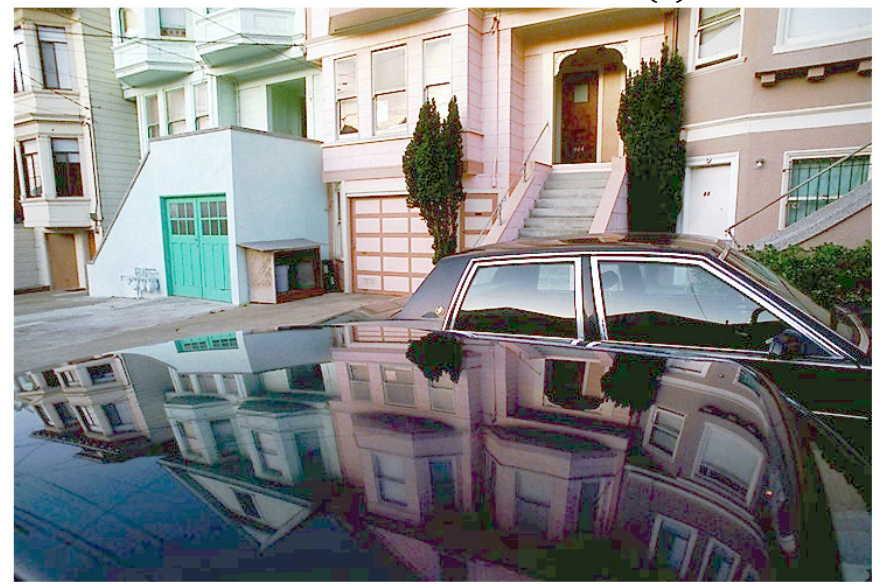

(c)

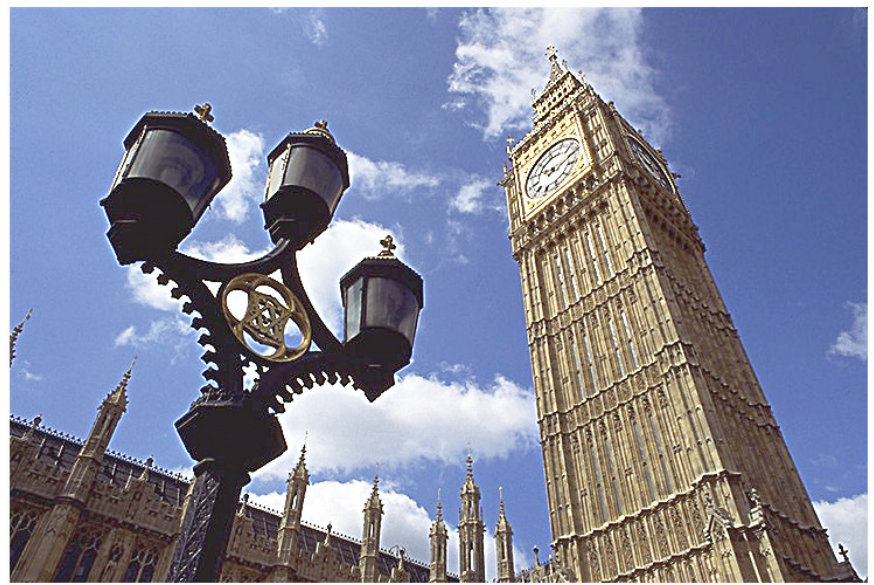

(a)

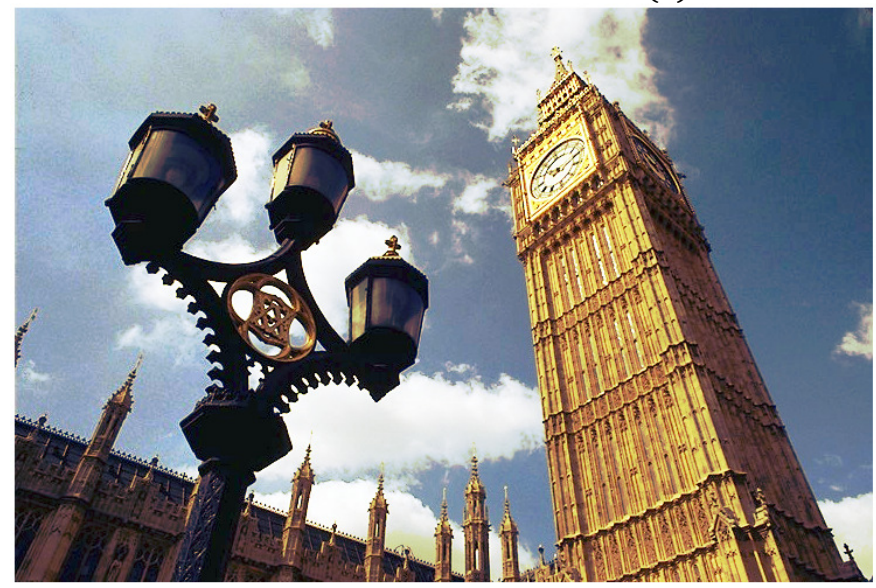

(c)

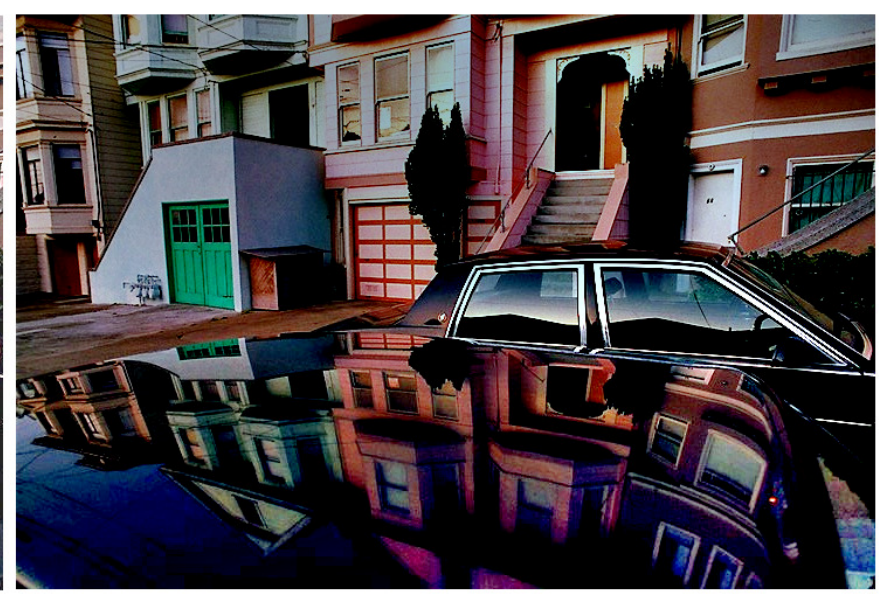

(b)

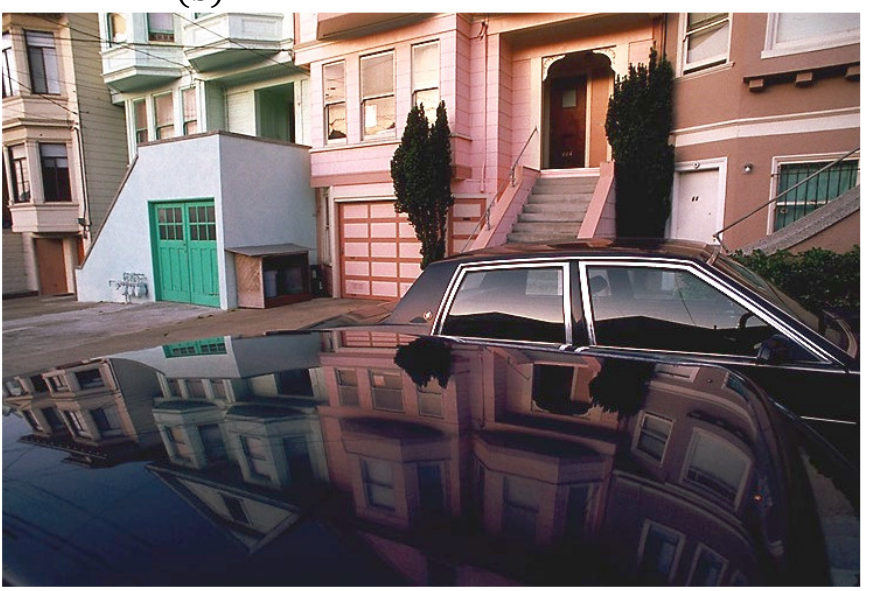

(d)

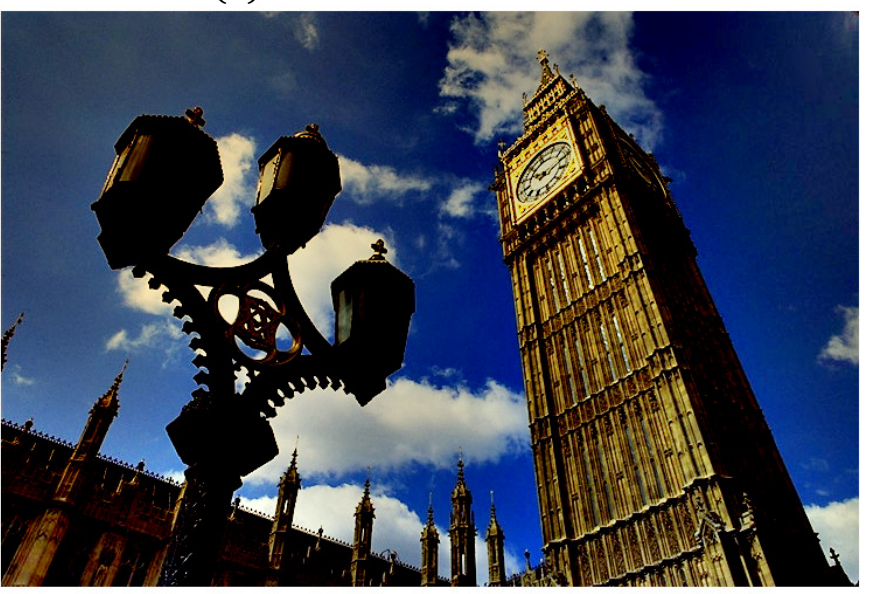

(b)

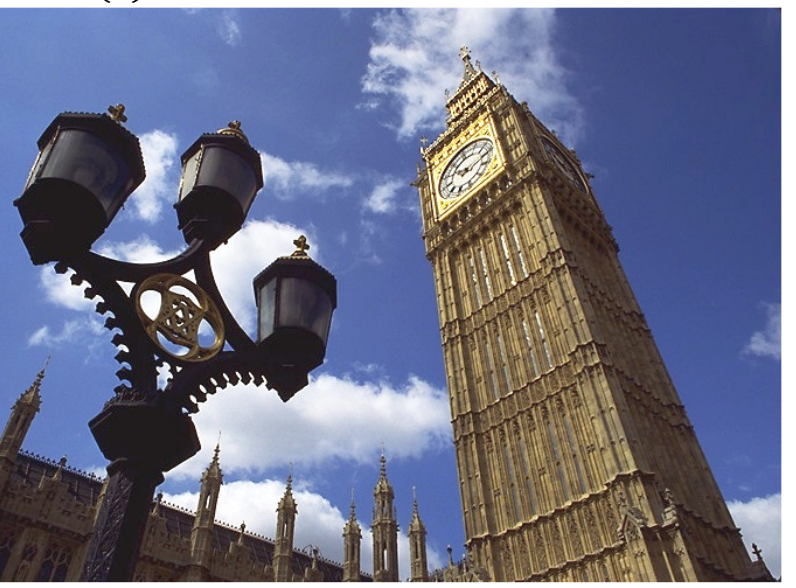

(d) 


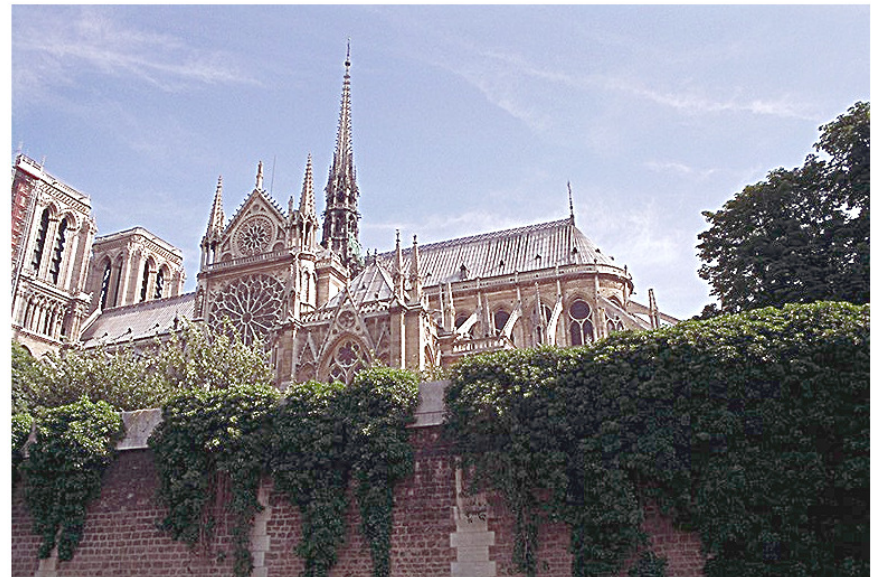

(a)

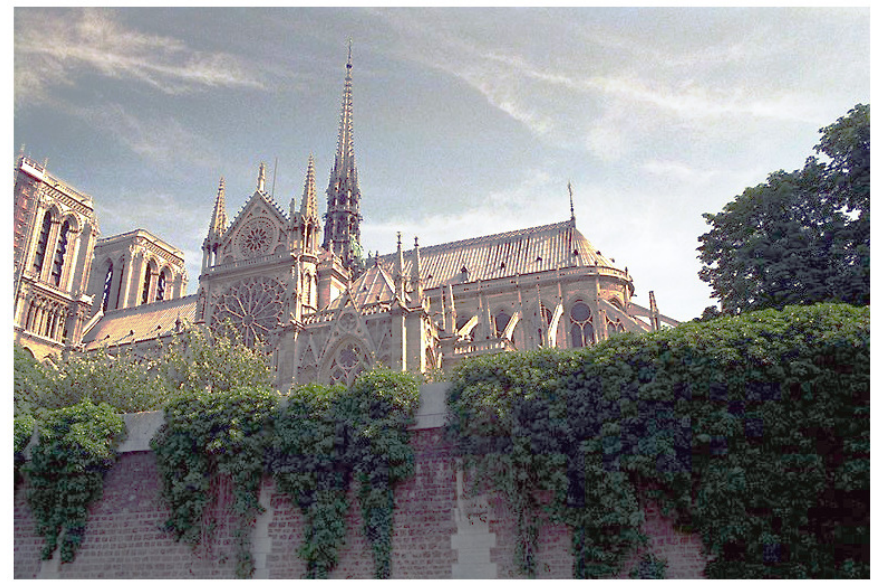

(c)

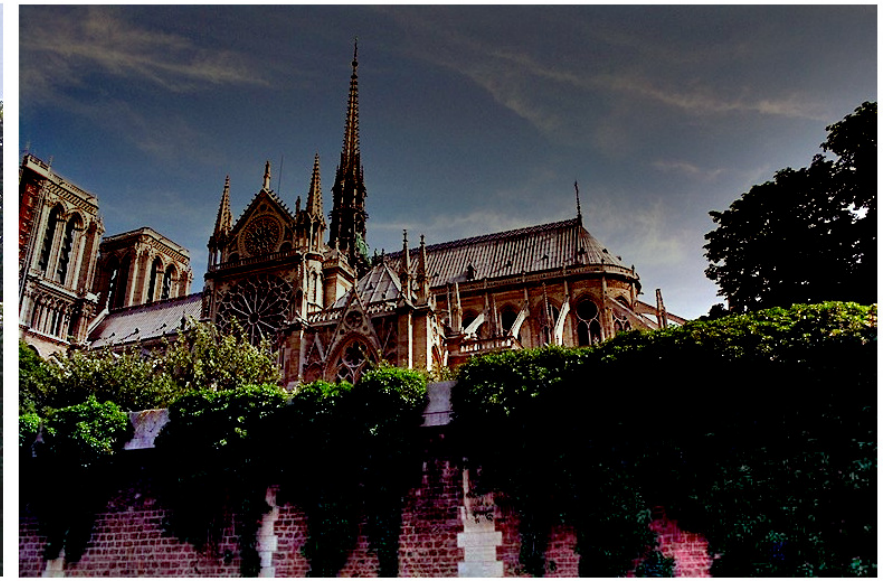

(b)

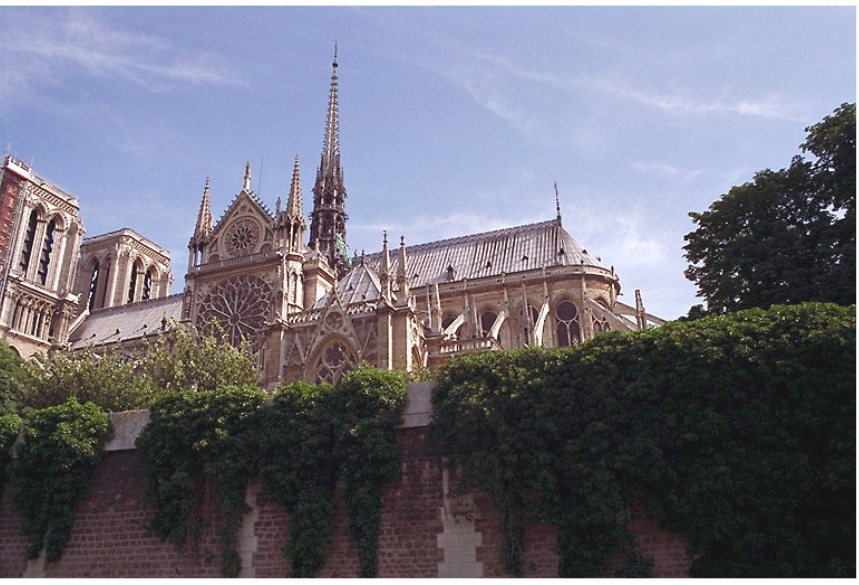

(d)

Figure 10 image enhanced with (a) Frequency domain Homomorphic filtering (b) MSRCR (c) Histogram Equalization (d) Fuzzy Homomorphic filter

Table 1. Fuzzy and crisp image statistics for shoe image

\begin{tabular}{cccccc}
\hline $\begin{array}{c}\text { Image } \\
\text { Measure }\end{array}$ & $\begin{array}{c}\text { Original } \\
\text { Image }\end{array}$ & MSRCR & $\begin{array}{c}\text { Freq } \\
\text { Homfilter }\end{array}$ & HE & $\begin{array}{c}\text { Fuzzy } \\
\text { Homfilter }\end{array}$ \\
\hline FE & 0.45 & 0.56 & 0.80 & 0.68 & 0.63 \\
LFI & 0.25 & 0.10 & 0.60 & 0.49 & 0.31 \\
QFI & 0.33 & 0.70 & 0.65 & 0.57 & 0.88 \\
RV & 1.00 & 14.75 & 2.19 & 5.85 & 19.78 \\
RSD & 1.00 & 3.84 & 1.48 & 2.42 & 4.45 \\
\hline
\end{tabular}

Table 2. Fuzzy and crisp image statistics for car image

\begin{tabular}{cccccc}
\hline $\begin{array}{c}\text { Image } \\
\text { Measure }\end{array}$ & $\begin{array}{c}\text { Original } \\
\text { Image }\end{array}$ & MSRCR & $\begin{array}{c}\text { Freq } \\
\text { Homfilter }\end{array}$ & HE & $\begin{array}{c}\text { Fuzzy } \\
\text { Homfilter }\end{array}$ \\
\hline FE & 0.79 & 0.51 & 0.70 & 0.66 & 0.77 \\
LFI & 0.62 & 0.07 & 0.53 & 0.49 & 0.60 \\
QFI & 0.67 & 0.66 & 0.61 & 0.57 & 0.65 \\
RV & 1.00 & 14.75 & 2.19 & 5.85 & 19.78 \\
RSD & 1.00 & 3.84 & 1.48 & 2.42 & 4.45 \\
\hline
\end{tabular}

Table 3. Fuzzy and crisp image statistics for tower clock image

\begin{tabular}{cccccc}
\hline $\begin{array}{c}\text { Image } \\
\text { Measure }\end{array}$ & $\begin{array}{c}\text { Original } \\
\text { Image }\end{array}$ & MSRCR & $\begin{array}{c}\text { Freq } \\
\text { Homfilter }\end{array}$ & HE & $\begin{array}{c}\text { Fuzzy } \\
\text { Homfilter }\end{array}$ \\
\hline FE & 0.80 & 0.49 & 0.69 & 0.66 & 0.78 \\
LFI & 0.62 & 0.05 & 0.52 & 0.48 & 0.60 \\
QFI & 0.67 & 0.69 & 0.60 & 0.57 & 0.66 \\
RV & 1.00 & 14.75 & 2.19 & 5.85 & 19.78
\end{tabular}

Nigerian Journal of Technology,

\begin{tabular}{cccccc} 
RSD & 1.00 & 3.84 & 1.48 & 2.42 & 4.45 \\
\hline \multicolumn{7}{c}{ Table 4. Fuzzy and crisp image } & statistics for cathedral image \\
\hline $\begin{array}{c}\text { Image } \\
\text { Measure }\end{array}$ & $\begin{array}{c}\text { Original } \\
\text { Image }\end{array}$ & MSRCR & $\begin{array}{c}\text { Freq } \\
\text { Homfilter }\end{array}$ & HE & $\begin{array}{c}\text { Fuzzy } \\
\text { Homfilter }\end{array}$ \\
\hline FE & 0.72 & 0.40 & 0.60 & 0.66 & 0.69 \\
LFI & 0.52 & 0.02 & 0.43 & 0.48 & 0.49 \\
QFI & 0.58 & 0.60 & 0.53 & 0.57 & 0.56 \\
RV & 1.00 & 14.75 & 2.19 & 5.85 & 19.78 \\
RSD & 1.00 & 3.84 & 1.48 & 2.42 & 4.45 \\
\hline
\end{tabular}

The higher the relative spread of the image, the more the contrast. With this measure, the relative degree of contrast enhancement can be determined. Looking at the results in Table I, it is observed that the fuzzy Homomorphic filter far outperforms the other algorithms with regards to higher relative contrast enhancement. Its fuzzy nature allows for easy adaptability due to the intuitiveness of the method. Also, the parameters measured can be used as objective values for optimization of the algorithm.

\section{CONCLUSION}

This paper has described the implementation of a fuzzy Homomorphic image enhancement system, 
which provides a novel and intuitive method of image enhancement. As with most fuzzy image enhancement algorithms, results depend on the degree and type of image distortion as well as the chosen membership functions. However, fuzzy domain methods are wellsuited to the subjective and imprecise problem of image enhancement. This allows the use of linguistic terms based on subjective reasoning and experience to be effectively utilized in fuzzy image processing techniques. Future implementation of the fuzzy Homomorphic algorithm will entail a rule-based system composed of the derivation of rules observed in the results of processed images, leading to a more adaptive system.

\section{REFERENCES}

[1] D.-Y. L. Wen-Hung Liao, "Homomorphic Processing Techniques for Near-Infrared Images," presented at IEEE International Conference on Acoustics/Speech and Signal Processing 2003.

[2] T. Q. Peli, T.; and "Homomorphic restoration of images degraded by light cloud cover," presented at Acoustics, Speech, and Signal Processing, IEEE International Conference on ICASSP'84, 1984.

[3] R. E. W. Rafael C. Gonzalez, Steven L. Eddins, Digital Image Processing Using MATLAB: Prentice Hall, 2004.

[4] J.-C. Pinoli, "A general comparative study of the multiplicative homomorphic, log-ratio and logarithmic image processing approaches," Signal Processing, vol. Volume 58, pp. 11 - 45, 1997.

[5] R. P. M. Mancuso, GG. Rizzotto, "A Fuzzy Filter for Dynamic Range Reduction and Contrast Enhancement," presented at IEEE World Congress on Computational Intelligence: 3rd IEEE Conference on Fuzzy Systems, 1994.

[6] V. K. Ming-Jung Seow; Asari, "Homomorphic Processing System and Ratio Rule for Colour Image Enhancement," presented at IEEE 2004 International Joint Conference on Neural Networks, 2004
[7] R. K. YoungSik Choi, "A Robust Approach to Image Enhancement Based on Fuzzy Logic," IEEE Transactions on Image Processing, vol. 6, pp. 808825, 1997.

[8] R. J. Ross, Fuzzy Logic with Engineering Applications: McGraw-Hill, 1995.

[9] A. B. Kiran R. Bhutani, "An application of fuzzy relations to image enhancement," Pattern Recognition Letters, vol. 16, pp. 901-909, 1995.

[10] J. M. Keller, "Fuzzy set theory in computer vision: A prospectus," Fuzzy Sets and Systems, vol. 90, pp. 177-182, 1997.

[11] A. Kandel, Fuzzy Expert Systems. CRC Press, 1992.

[12] H. X. H.D. Cheng, "A novel fuzzy logic approach to contrast enhancement," Pattern Recognition, vol. 33, pp. 809-819, 2000.

[13] G. K. H. R. Tizhoosh, B. Michaelis, "On Fuzzy Enhancement of Megavolt Images in Radiation Therapy," presented at IEEE Conference on Fuzzy Systems, FUZZ-1EEE'97, Barcelona, Spain, 1997.

[14] M. N. Etienne E. Kerre, Fuzzy Techniques in Image Processing. Physica-Verlag, 2000.

[15] M. G. K. Delac, T. Kos, "Sub-Image Homomorphic Filtering Technique for Improving Facial Identification under Difficult Illumination Conditions," presented at International Conference on Systems, Signals and Image Processing, Budapest, Hungary, 2006.

[16] D. J. M. Hanmandlu, Rochak Sharma, "Color image enhancement by fuzzy intensification," Pattern Recognition Letters, vol. 24, pp. 81-87, 2003.

[17] Z.-u. R. Danial J. Jobson, Glenn A. Woodell, "A Multiscale Retinex for Bridging the Gap Between Color Images and the Human Observation of Scenes," IEEE Transactions on Image Processing, vol. Vol. 6, pp. 965-976, 1997.

[18] D. J. M. Hanmandlu, Rochak Sharma, "An Optimal Fuzzy System for Colour Image Enhancement," IEEE Transactions on Image Processing, vol. 15, pp. 29562966, 2006. 